\title{
Cephalad Angulation of Epidural Needle Insertion May Be an Important Factor for Safe Epidural Space Approach: a Mathematical Model
}

\author{
Satoki Inoue ${ }^{1}$, Masahiko Kawaguchi ${ }^{2}$, Hitoshi Furuya ${ }^{3}$
}

\begin{abstract}
Summary: Inoue S, Kawaguchi M, Furuya H - Cephalad Angulation of Epidural Needle Insertion May Be an Important Factor for Safe Epidural Space Approach: a Mathematical Model.

Background and objectives: Epidural approach angle is one of the determining factors for needle traveling length to reach the epidural space. Likewise, the margin of safety against accidental dural puncture should be affected by epidural approach angle because the space available for epidural needle tip movement in the epidural space is depending on the inserting angle to the epidural space. The purpose of this study is to conjecture the effect of the angle formed with the skin and an epidural needle on the margin of safety against accidental dural puncture using a mathematical model.

Case report: Suppose that the width of the epidural space is $A \mathrm{~mm}$ and that the force advancing the epidural catheter is $\mathrm{C}$ kgf and the diameter of the catheter is $D \mathrm{~mm}$. In this situation, assuming that the cephalad angle with the midline approach formed with the skin and an epidural needle is $\theta$, the following parameters can be determined: the available distance for the needle tip in the epidural space $=A / \sin \theta \mathrm{mm}$; cephalad advancing force of catheter $=C^{*} \cos \theta \mathrm{kgf}$; and the pressure at the site of dura matter where the epidural catheter is pushing $=400^{*} \mathrm{C}^{*} \sin \theta \cdot \pi \mathrm{D}^{-2} \mathrm{kgf} . \mathrm{cm}^{-2}$. The longer the distance available for the needle tip, the larger the margin of safety for dura injury by the epidural needle. It is supposed that $\theta$ should be set smaller to make the margin of safety larger. Likewise, smaller $\theta$ could decrease the pushing pressure and more effectively create a distinct cephalad advancing force.
\end{abstract}

Conclusions: Acute cephalad angulation should be considered to increase the margin of safety for epidural approach and catheterization.

Keywords: Epidural Space; Anesthesia, Epidural; Models, Theoretical; Safety Management.

\section{INTRODUCTION}

The rate of accidental dural puncture after epidural approach has been estimated and varying from $0.19 \%$ to $3.6 \%$ in several studies ${ }^{1-4}$. Once accidental dural puncture occurs it has been suggested that postdural puncture headache (PDPH) develops in more than $50 \%$ of these patients, which can cause significant morbidity ${ }^{5}$. Several prevention methods against PDPH have been proposed, some of them may be promising options to prevent PDPH ${ }^{6}$. However, decreasing the rate of accidental dural puncture is supposed to be a more promising option rather than any existing prevention methods for PDPH.

Received from Department of Anesthesiology, Nara Medical University (NMU), Japan

1. MD; Assistant Professor

2. MD; Associate Professor, Nara Medical University, Japan

3. MD; Professor, Nara Medical University, Japan

Submitted on March 16, 2011.

Approved on April 4, 2011.

Correspondence to:

Dr. Satoki Inoue

奈良県立医科大学 (Nara Medical University)

840 Shijo-cho Kashihara, Nara 634-8522, Japan

E-mail: seninoue@naramed-u.ac.jp
It is obvious that epidural approach angle is one of the determining factors for needle traveling length to reach the epidural space. Likewise, the margin of safety against accidental dural puncture should be affected by epidural approach angle because space available for epidural needle tip movement in the epidural space is depending on the inserting angle to the epidural space. The purpose of this study is to conjecture the effect of the angle formed with the skin and an epidural needle on the margin of safety against accidental dural puncture using a mathematical model.

\section{MATHEMATICAL MODEL}

First, suppose that the width of the epidural space is A mm and that the subcutaneous distance from the skin surface to the epidural space is $B \mathrm{~cm}$. In addition, assume that the force advancing the epidural catheter is $\mathrm{C}$ kgf and the diameter of the catheter is $\mathrm{D} \mathrm{mm}$. In this situation, assuming that the cephalad angle with the midline approach formed with the skin and an epidural needle is $\theta$, the following parameters can be determined (Figure 1). Here, it is not supposed that the caudad angulation is used as another option.

1. The available distance for the needle tip in the epidural space $=A / \sin \theta \mathrm{mm}$ 


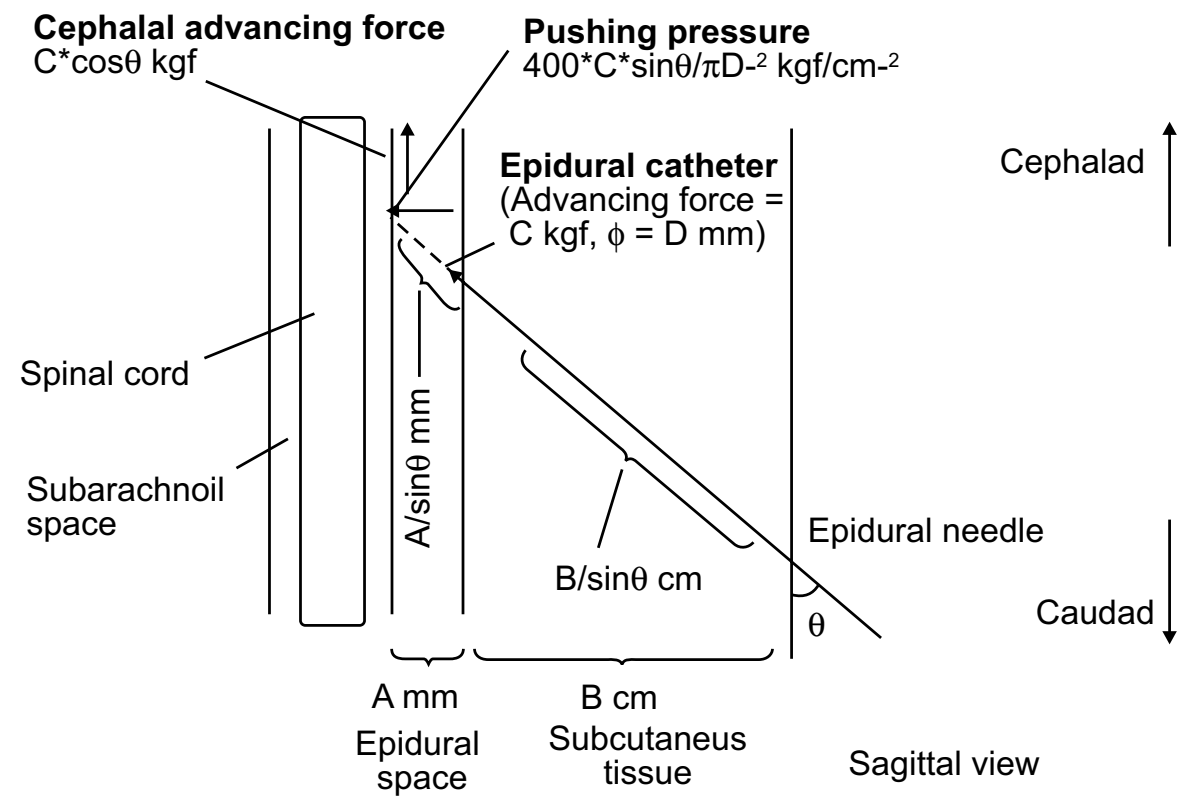

Figure 1 - Epidural Approach Model in the Sagittal View.

The width of the epidural space is A mm. The subcutaneous distance from the skin surface to the epidural space is $B \mathrm{~cm}$. The force advancing the epidural catheter is $C$ kgf. The diameter of the catheter is $D \mathrm{~mm}$. The angle formed with the skin and an epidural needle is $\theta$. The available distance for the needle tip in the epidural space $=\mathrm{A} / \sin \theta \mathrm{mm}$. The distance from the insertion point to the reaching point at the epidural space $=$ $\mathrm{B} / \sin \theta \mathrm{cm}$. Cephalad advancing force of catheter $=\mathrm{C}^{*} \cos \theta \mathrm{kgf}$. The pressure at the site of the dura matter where the epidural catheter is pushing $=400^{*} \mathrm{C}^{*} \sin \theta \cdot \pi \mathrm{D}^{-2} \mathrm{kgf} \cdot \mathrm{cm}^{-2}$.

2. The distance from the insertion point to the reaching point at the epidural space $=\mathrm{B} / \mathrm{sin} \theta \mathrm{cm}$

3. Cephalad advancing force of catheter $=C^{*} \cos \theta \mathrm{kgf}$

4. The pressure at the site of the dura matter where the epidural catheter is pushing $=400^{*} \mathrm{C}^{*} \sin \theta \cdot \pi \mathrm{D}^{-2} \mathrm{kgf} . \mathrm{cm}^{-2}$

\section{COMMENTS}

Even at the moment the epidural needle tip reaches the epidural space, the needle tip can be advanced to some degree in spite of paying the greatest attention to prevent injury of the dura. Therefore, the longer the distance available for the needle tip, the larger the margin of safety for dura injury by the epidural needle can become (Figure 1). It is supposed that should be set smaller to make the margin of safety larger.

The epidural needle cephalad direction may be limited because of the bony configuration of the spine when midline approach is used, especially in case of lumbar epidural approach. Paramedian approach can provide a wide range of angles for epidural needle direction. Therefore, paramedian approach may be more suitable to set the cephalad angulation smaller. Actually, it has been suggested that the incidence of accidental dural puncture is lower when using the paramedian approach based on the study using epiduroscopy in cadav- ers $^{7}$. In addition, a clinical study showed that the paramedian approach was associated with a lower frequency of technical problems compared to the midline approach ${ }^{8}$. These reported results might be attributed to the steeper angle in the paramedian approach. Take the case of needle direction at 45 degrees, it could increase the margin of safety against accidental dural puncture by 1.4 -fold compared with vertical direction (Figure 1).

Incidentally, advancement of the epidural catheter can be effectively performed when is set small because the force advancing the epidural catheter is greatly directed toward cephalad in such conditions. There are several articles available showing that epidural catheterization using paramedian approach is associated with fast insertion and minimum risk of catheter coiling 9-11. These researchers have also suggested that fast, safe, and effective catheterization may be attributed to paramedian approach with cephalad angulation. Recently, it has been suggested that the spinal dura matter is also sensitive to traction and stretch, which might be one of the causes of paresthesia during epidural catheterization ${ }^{12}$. The pushing pressure is also reduced by acute cephalad angulation, resulting in reduction of dural stretch (Figure 1). It might be a possible reason for reducing paresthesia during epidural catheterization using paramedian approach ${ }^{12,13}$. Although a recent report denies that catheter passage can 
occur in the presence of intact dura, stronger pushing pressure would let the catheter pass the dura in the presence of dural damage with the epidural needle ${ }^{14}$. In such a situation, steeper cephalad angulation would provide weaker pushing pressure against the dura, which might reduce unintentional intrathecal catheterization. Similar to the previous paragraph, take the case of needle direction at 45 degrees compared with vertical direction, it could decrease the pushing pressure to the dura by 0.7 -fold compared with vertical direction. In this condition, a noteworthy effect is that it could create a distinct cephalad advancing force that unlike the advanced direction cannot be determined at vertically directed catheterization (Figure 1). In clinical situations, this is not completely related to the above hypothesis because Tuohy needles are used, which can determine the direction of catheterization.

In clinical settings, it might be difficult to verify the validity of this proposed theory based on the mathematical model because of the nature of its low rates of incidence. For the statistical analysis, very large study population would be required to investigate this hypothesis as a randomized clinical trial. Besides, approach methods for the epidural needle insertion surely depend on the attending anesthesiologists' preference. This may imply that it is nearly impossible to compare several approach methods on equal terms. For these reasons, it is considered suitable to propose this hypothesis using this mathematical model.

In conclusion, the smaller the angle formed with the skin and an epidural needle becomes, the larger the margin of safety for epidural space approach can be. In addition, with this condition epidural catheterization would be safer and easier to advance toward cephalad direction. Therefore, the paramedian approach with steep cephalad angulation should be considered to increase the margin of safety for epidural approach and catheterization. 